\title{
Role of prothrombotic polymorphisms in successful or unsuccessful aging
}

\author{
Loredana Vaccarino • Giusi Irma Forte • Marisa Palmeri - Gabriella Misiano • \\ Elisa Porcellini - Martina Chiappelli $\cdot$ Letizia Scola $\cdot$ Calogero Caruso • \\ Federico Licastro · Domenico Lio
}

Received: 27 January 2011 / Accepted: 4 March 2011

(C) Springer Science+Business Media B.V. 2011

\begin{abstract}
The study of the genetic profile of centenarians aims to identify the genes and allelic variants which may influence a greater life expectancy and that can be considered as predisposing factors associated to the aging diseases, such as Alzheimer. Centenarians, that represent a cohort of selected survivors, show an hypercoagulability state characterised by striking signs of high coagulation enzyme activity, as directly assessed by the tested higher plasma level of some important factors involved in the haemostasis balance. Anyway, these individuals seem to have a reduced susceptibility to dementia, as well as to cardiovascular events. In this study we analyze the frequencies of Leiden Factor V polymorphism (G1691A), and G20210A of prothrombin (FII) in three cohorts of subjects: patients with Alzheimer's disease (unsuccessful aging), nonagenarians (successful aging) and young healthy controls, to assess whether allelic variants associated to the modification of haemostatic system function, may play a role in the protection or susceptibility to
\end{abstract}

L. Vaccarino · G. I. Forte $(\bowtie) \cdot$ M. Palmeri .

G. Misiano $\cdot$ L. Scola $\cdot$ C. Caruso $\cdot$ D. Lio

Department of Biopathology and Medical and Forensic

Biotechnology, University of Palermo,

Corso Tukory 211, 90134 Palermo, Italy

e-mail: giusiforte@unipa.it

E. Porcellini · M. Chiappelli · F. Licastro

Department of Sperimental Pathology,

University of Bologna, Bologna, Italy
Alzheimer disease, as well as to reach a successful aging. No significant differences were observed in the frequencies of the three groups studied. These results indicate that the presence or absence of the gene variants examined did not influence the achievement of advanced age and are not risk factors for Alzheimer's disease. The state of hypercoagulability and the possession of these risk alleles appear to be compatible with the achievement of longevity and are not implied as risk factors in Alzheimer disease development.

Keywords Leiden factor V · Prothrombin . Nonagenarians $\cdot$ Alzheimer disease $\cdot$ Aging

\section{Introduction}

Aging is characterised by a progressive loss of ability to effectively interact with the environmental stimuli and it is accompanied by progressive modification and adaptations that are influenced by lifestyle and genetic background of the single individual, conditioning the reaching of a successful or an unsuccessful ageing. The preservation of cognitive functioning is an essential component of successful aging. The ability to distinguish those individuals who maintain cognitive health into advanced age from those who will develop a cognitive decline may influence public health efforts, in order to prevent or delay the onset of cognitive impairment in old age. 
There is a growing evidence implicating vascular risk factors and related subclinical cerebrovascular damage in cognitive impairment and dementia, but the role of inflammation in vascular and neurodegenerative processes is poorly understood. In the Alzheimer Disease (AD), and in other neurodegenerative diseases as well, it is hypothesized that an alteration of the neurovascular unit, which is the functional unit encompassing vascular cells, astrocytes and perivascular neurons, is an early event in the pathogenesis of the disease (Iadecola 2004).

Dysfunction of the neurovascular unit results in an impaired blood brain barrier functions, dysregulation of cerebral blood flow and impairment of betaamyloid (A-beta) clearance with increase of oligomers and soluble A-beta forms (Zlokovic 2008).

In this view, it might be of some interest the hypothesis that one of the constant feature of ageing, the progressive increase of concentrations of some coagulation factors, such as factor VII, factor VIII and fibrinogen, the increase of platelets activity and fibrinolysis impairment, might play a role in the well known age associated increased susceptibility to cardiovascular and thromboembolic diseases, as well as, in the pathogenesis of dementia. Some inherited prothrombotic variants of coagulation proteins, such as factor V (FV) Leiden and prothrombin G20210A polymorphism, may play a key role in this scenario.

Leiden Factor V is present in $20 \%$ of patients with venous thrombosis and in over half of probands in selected families with thrombophilia. This means that it represents the most common genetic abnormality in patients with thrombosis (Rosendaal et al. 1995).

In particular, the heterozygous form of Leiden factor $\mathrm{V}$ increases the risk of thrombosis $3-8 \mathrm{x}$, while the homozygous form presents a risk which may be up to 80 times greater (Koster et al. 1993; Ridker et al. 1995; Anderson et al. 1991). Furthermore, the mutation $\mathrm{G} \rightarrow \mathrm{A}$ at the position 2010 in the $3^{\prime}$-untranslated region of the prothrombin gene is associated with an increased level of prothrombin and such an increased risk of developing thrombosis (Poort et al. 1996). In spite of the potential role that prothrombotic variants of genes, coding for the coagulation factors, might play in Alzheimer's disease (AD) susceptibility (Gupta et al. 2005; Cortes-Canteli et al. 2010), very few studies have been performed on the association between coagulation gene polymorphisms and $\mathrm{AD}$ (Gerardino et al. 2006).
On the other hand, centenarians, that represent a cohort of selected survivors who benefit of a delay in the onset of diseases, that often cause mortality in the general population at significantly younger ages (Candore et al. 2006), show an hypercoagulability characterised by striking signs of high coagulation enzyme activity, as assessed directly by measuring factor VIIa plasma level or, indirectly, by measuring the peptides released following the activation of prothrombin, or factors IX and X, both well documented (Mari et al. 2008). However, these individuals seem to have a reduced susceptibility to dementia, as well as to cardiovascular events (Franceschi et al. 1995).

Considering the role that a genetically determined hypercoagulability might play in the cognitive decline associated with $\mathrm{AD}$, we analyzed the frequencies of polymorphisms G1691A of Factor V and G20210A of prothrombin (FII) in patients with $\mathrm{AD}$ and in subjects of age $>90$ years old, which having overcame the risk of ADs, might be considered the better model to assess whether these allelic variants are associated to the modification of haemostatic system function and if they have a role in the protection or susceptibility to the onset of AD. In addition, allele and genotype frequencies of patients and nonagenarians have been compared to those ones of a group composed by young healthy controls, in order to evaluate the possible effect of age stratification on genetic frequencies modification for the analysed genes.

\section{Materials and methods}

Subjects

This study included three groups of subjects.

AD patients 95 subjects (Northern Italy; mean age 70.3; mean onset age 68.9; 23 males and 72 females) affected by AD. Diagnosis of probable AD had been performed according to standard clinical procedures. The DMS IV and NINCDS-ADRDA criteria were applied to obtain the clinical diagnosis of $\mathrm{AD}$ (Mckhann et al. 1984). Cognitive performances and alterations were measured according to the Mini Mental State Evaluation (MMSE) and the Global Deterioration Scale (GDS) (Doody et al. 2001). All $\mathrm{AD}$ cases were defined as sporadic because the family history did not mention any first-degree relative with 
dementia. Age at onset was defined as the age when memory loss was first noticed by relatives, besides, 79 of $\mathrm{AD}$ patients showed clinical onset of the disease after 65 years of age (late-onset $\mathrm{AD}, \mathrm{LOAD}$ ) and 16 before this age (early onset AD, EOAD).

Nonagenarians. A group of 106 individuals of age $>90$ years old, matched for ethnicity and gender (Northern Italy; 21 males and 85 females) with control subjects. Cognitive status of these subjects was assessed by administration of the Mini-Mental State Examination (Mean score $28 \pm 2$; score for inclusion as normal control subjects $>24$ ) (Cockrell and Folstein 1988).

Young subjects. A group composed by 65 healthy subjects aged between 20 and 50 years old matched for ethnicity and gender (Northern Italy; 18 males and 47 females) with nonagenarians subject;

Written informed consent for the study's enrolling and for personal data management was obtained from controls and relatives of nonagenarians and $\mathrm{AD}$ patients according to Italian laws.

Blood specimens were collected in tripotassium EDTA sterile tubes, DNA extracted (Miller et al. 1988) and processed for genotyping as described below.

\section{G1691A of FV and 20210GA of FII allele discrimination}

The two polymorphisms: G1691A (FV Leiden) and G20210A were typed by two pre-made Taqman RealTime PCR allelic discrimination assays, by Applied Biosystems.

Briefly, $10 \mathrm{ng}$ of DNA for each sample were used in a PCR reaction, containing $1 \times$ optimized master mix and $1 \times$ specific primers/probes mix assay, according to manufactory protocol in a final volume of $25 \mu 1$.

For each polymorphism, two identical probes, except for the central nucleotide that specifically recognizes the single nucleotide polymorphism (SNP) were used, each one labelled at the $5^{\prime}$ extremity with different dyes, FAM and VIC in this case and at the $3^{\prime}$ extremity with a quencher dye, that in this case was the Minor Groove Binder (MGB) dye.

Then, the amplification was performed in 7300 Real-Time ABI Prism PCR System (Applied Biosystems, USA), using a standard amplification protocol
( 1 cycle of $2^{\prime}$ at $50^{\circ} \mathrm{C} ; 1$ cycle of $10^{\prime}$ at $95^{\circ} \mathrm{C}$ and 40 cycles of $15^{\prime \prime}$ at $95^{\circ} \mathrm{C}$ plus $15^{\prime \prime}$ at $60^{\circ} \mathrm{C}$ ), and the results were available in the report sheet of 7300 System SDS v1.3 Software.

Moreover, samples were graphically grouped in 3 genotypic clusters, easily recognizable in the Allelic Discrimination plot on the basis of the two probe's fluorescence intensity emissions, whereas the uncertain cases were also evaluated for the grow up of the fluorescence emission curve of each dyes on the components sheet.

Statistical analysis

The data were tested for goodness of fit between the observed and expected genotype $(3 \times 2$ tables $)$ and allele $(2 \times 2$ tables $)$ frequencies. Fisher's exact tests were performed to calculate significant different genotype or allelic distributions between AD patients, nonagenarians and matched controls. It was accepted a significance with $P$ values $<0.05$. The HardyWeinberg equilibrium was tested using the Pearson test.

\section{Results and discussion}

The goal of the present study is to assess whether allelic variants, G1691A Factor V (Leiden FV) and G20210A prothrombin single nucleotide polymorphisms (SNP), associated to the modification of haemostatic system function, may play a role in the protection or susceptibility to $\mathrm{AD}$, as well as to reach a successful aging.

Table 1 shows the genotypes and alleles frequencies for Leiden FV in our sample of 95 patients with AD, 106 nonagenarians and 65 healthy young subjects.

No significant differences were observed in the genotype and allele frequencies among the three groups studied $(P>0.05)$. However, we observed a slight increase of the mutated allele frequency, due to an increase of the heterozygosis condition in the elderly and in the AD patients, where the mutated allele is more common in comparison with young individuals.

Mari et al. (1996), previously described similar allele frequency for this mutation in Italian centenarians without any history of thrombotic events, also in 
Table 1 FV $1691 \mathrm{G} \rightarrow$ A (FV LEIDEN) Genotypic and allelic frequencies of polymorphisms G1691A of Factor V in 95 patients with $\mathrm{AD}, 106$ nonagenarians and 65 healthy young subjects

\begin{tabular}{|c|c|c|c|c|c|c|}
\hline & \multirow[t]{2}{*}{$N$} & \multicolumn{3}{|c|}{ Genotypic frequencies } & \multicolumn{2}{|c|}{ Allelic frequencies } \\
\hline & & $\mathrm{G} / \mathrm{G}$ & G/A & $\mathrm{A} / \mathrm{A}$ & G & A \\
\hline Alzheimer's & 95 & $95.8 \%(91)$ & $4.2 \%(4)$ & $0 \%(0)$ & $97.9 \%(186)$ & $2.1 \%(4)$ \\
\hline$>90$ years old subjects & 106 & $96.2 \%(102)$ & $3.8 \%(4)$ & $0 \%(0)$ & $98.1 \%(208)$ & $1.9 \%(4)$ \\
\hline Young subjects & 65 & $96.9 \%(63)$ & $3.1 \%(2)$ & $0 \%(0)$ & $98.5 \%(128)$ & $1.5 \%(2)$ \\
\hline
\end{tabular}

five centenarians carrying the factor $\mathrm{V}$ mutation, exposed during their life to circumstantial risk factors for thrombosis.

Moreover, studies on octogenarians and centenarians in Europe suggest a normal life span expectancy for Leiden FV carriers. In 3 studies of people older than 80 years old, living in Italy (Mari et al. 1996), Denmark (Rees et al. 1997) and the United Kingdom (Kristensen et al. 1998), the prevalence of the Leiden FV gene in the elderly population was similar to its prevalence in the general population. If Leiden FV carriers were dying at a younger age for VTE events, then the expected prevalence of the FV Leiden allele in an older age group should be lower. In a population based mortality study of octogenarians living in Leiden, 660 patients were observed for 10 years. The relative risk of mortality in Leiden FV carriers was identical to that one observed in the no carriers (Heijmans et al. 1998).

Our results seem to confirm the above mentioned data and demonstrate that Leiden FV variant seems do not play a role as susceptibility factor for AD.

Similar results were obtained typing the prothrombin G20210A polymorphism. The genotypic and allelic distribution of the single nucleotide polymorphism (SNP), located at the position G 20210A of the prothrombin (FII) gene, shows no statistical differences between young subjects, nonagenarians and patients with $\mathrm{AD}$ (Table 2).
As well known, a mutation in the $3^{\prime}$ untranslated region of the prothrombin gene $(\mathrm{G} 20210 \mathrm{~A})$, associated with higher plasma levels of prothrombin, is a common genetic risk factor for venous thrombosis (Poort et al. 1996). In addition several studies have reported an association of Leiden Factor V (FV) and prothrombin (FII) G20210A with arterial thrombotic disorders, especially in the association with the juvenile cases (Kim and Becker 2003).

As a paradox, in long-living subjects the presence of high-risk alleles, sometimes detected in the homozygous form, for many polymorphisms related to the cardiovascular diseases, has been documented (Mari et al. 1996). In particular, in some studies centenarians showed a paradoxically significantly higher frequency of the high-risk genetic markers Leiden factor V and G20210A mutation in prothrombin gene, than young individuals (Mannucci et al. 1997; Sacchi et al. 1999), although they demonstrate to be able in the escaping from the onset of the major age-related diseases as AD.

In conclusion, all together our results indicate that the state of hypercoagulability and the possession of several high-risk alleles and well-known atherothrombotic risk markers appear to be compatible with longevity and are not implied as risk factor in $\mathrm{AD}$ development. However, considering the relative low number of subjects analyzed in our study, these results need to be confirmed in larger cohorts of subjects to

Table 2 FII $20210 \mathrm{G} \rightarrow$ A Genotypic and allelic frequencies of polymorphism G20210A of prothrombin gene observed in 95 patients with $\mathrm{AD}, 106$ nonagenarians and 65 healthy young subjects

\begin{tabular}{|c|c|c|c|c|c|c|}
\hline & \multirow[t]{2}{*}{$N$} & \multicolumn{3}{|c|}{ Genotypic frequencies } & \multicolumn{2}{|c|}{ Allelic frequencies } \\
\hline & & $\mathrm{G} / \mathrm{G}$ & G/A & $\mathrm{A} / \mathrm{A}$ & G & A \\
\hline Alzheimer's & 95 & $94.7 \%(90)$ & $5.3 \%(5)$ & $0 \%(0)$ & $97.4 \%(185)$ & $2.6 \%(5)$ \\
\hline$>90$ years old subjects & 106 & $95.3 \%(101)$ & $4.7 \%(5)$ & $0 \%(0)$ & $97.6 \%(207)$ & $2.4 \%(5)$ \\
\hline Young subjects & 65 & $96.9 \%(63)$ & $3.1 \%(2)$ & $0 \%(0)$ & $98.5 \%(128)$ & $1.5 \%(2)$ \\
\hline
\end{tabular}


definitively exclude that Leiden Factor V G169A and/ or Prothrombin G20210A polymorphisms might have a role in successful or unsuccessful aging.

On the other hand, it is well known that the protection or susceptibility effect of a single polymorphism might be modulate both as additive or synergic and antagonistic influence by the presence of other polymorphisms in genes involved in the same metabolic or functional pathway. In this view, the interaction of the analysed polymorphisms with other functionally relevant genetic variants of coagulation and of the strictly related complement cascade should be considered in future studies. As reviewed by Amara et al. (2008) both cascades contain series of serine-proteases with evidence of some shared activators and inhibitors. Factor XIIa is able to activate $\mathrm{C} 1 \mathrm{q}$, and thereby the classical pathway of complement. Besides thrombin, generated at inflammatory sites in response to complement activation, is involved in a negative feedback loop preventing thrombosis during inflammation. Finally the complement activation product $\mathrm{C} 5 \mathrm{a}$ has also been reported to induce tissue factor (TF) activity and may activate the exogenous (TF-dependent) coagulation pathway. This seems to be more relevant considering that two independent genome-wide association studies (Lambert et al. 2009; Seshadri et al. 2010) have recently demonstrated that the rs6656401 SNP of the complement component $(3 \mathrm{~b} / 4 \mathrm{~b})$ receptor 1 influencing a reduced clearance of complement-opsonized A $\beta 42$ immune complexes might be involved as a major genetic risk factor for late onset AD. So an increased complement activity due to circulating IC persistence might enhance coagulation cascade trough the complex interaction summarised above.

Acknowledgments This research was supported by Grants from the Ministry of Education, University and Research (Cofin to D.L. and local initiatives to D.L.), and from Ministry of Health. G.I.F. is a $\mathrm{PhD}$ researcher, working on a project funded by a grant received by Italian Ministry of Education, University and Research, and this work represents an implementation of her own final report.

\section{References}

Amara U, Rittirsch D, Flierl M, Bruckner U, Klos A, Gebhard F, Lambris JD, Huber-Lang M (2008) Interaction between the coagulation and complement system. Adv Exp Med Biol 632:71-79
Anderson FA, Wheeler HB, Goldberg RJ, Hosmer DW, Patwardhan NA, Jovanovic B, Forrier A, Dalen JE (1991) A population based perspective of the hospital incidence and case-fatality rates of deep vein thrombosis and pulmonary embolism. The Worcester DVT study. Arch Intern Med 151:933-938

Candore G, Balistreri CR, Listi F, Grimaldi MP, Vasto S, Colonna-Romano G, Franceschi C, Lio D, Caselli G, Caruso C (2006) Immunogenetics, gender, and longevity. Ann N Y Acad Sci 1089:516-537

Cockrell JR, Folstein MF (1988) Mini-mental state examination (MMSE). Psychopharmacol Bull 24:689-692

Cortes-Canteli M, Paul J, Norris EH, Bronstein R, Ahn HJ, Zamolodchikov D, Bhuvanendran S, Fenz KM, Strickland S (2010) Fibrinogen and beta-amyloid association alters thrombosis and fibrinolysis: a possible contributing factor to Alzheimer's disease. Neuron 66:695-709

Doody RS, Massman P, Dunn JK (2001) A method for estimating progression rates in Alzheimer's disease. Arch Neurol 58:449-454

Franceschi C, Monti D, Sansoni P, Cossarizza A (1995) The immunology of exceptional individuals "the lesson of centenarians". Immunol Today 16:12-16

Gerardino L, Papaleo P, Flex A, Gaetani E, Fioroni G, Pola P, Pola R (2006) Coagulation factor XIII Val34Leu gene polymorphism and Alzheimer's disease. Neurol Res 28:807-809

Gupta A, Watkins A, Thomas P, Majer R, Habubi N, Morris G, Pansari K (2005) Coagulation and inflammatory markers in Alzheimer's and vascular dementia. Int J Clin Pract 59:52-57

Heijmans BT, Westendorp RG, Knook DL (1998) Risk of mortality and the factor V Leiden mutation in a population-based cohort. Thromb Haemost 80:607-609

Iadecola C (2004) Neurovascular regulation in the normal brain and in Alzheimer's disease. Nat Rev Neurosci $5: 347-360$

Kim RJ, Becker RC (2003) Association between factor V Leiden, prothrombin G20210A, and methylenetetrahydrofolate reductase C677T mutations and events of the arterial circulatory system: a meta-analysis of published studies. Am Heart J 146:948-957

Koster T, Rosendaal FR, De Ronde H, Briet E, Vandenbroucke JP, Bertina RM (1993) Venous thrombosis due to a poor anticoagulant response to activated protein $\mathrm{C}$ : Leiden thrombophilia study. Lancet 342:1503-1506

Kristensen SR, Andersen-Ranberg K, Bathum L, Jeune B (1998) Factor V Leiden and venous thrombosis in Danish centenarians. Thromb Haemost 80:860-861

Lambert JC, Heath S, Even G et al (2009) Genome-wide association study identifies variants at CLU and CR1 associated with Alzheimer's disease. Nat Genet 41:1094-1099

Mannucci PM, Mari D, Merati G, Tagliabue L, Sacchi E, Taioli E, Sansoni P, Bertolini S, Franceschi C (1997) Gene polymorphisms predicting high plasma levels of coagulation and fibrinolysis proteins. A study in centenarians. Arterioscler Thromb Vasc Biol 17(4):755-759

Mari D, Duca F, Mannucci PM, Franceschi C (1996) Mutant factor V (Arg506Gln) in healthy centenarians. Lancet $347: 1044$ 
Mari D, Coppola R, Provenzano R (2008) Hemostasis factors and aging. Exp Gerontol 43(2):66-73

Mckhann G, Drachman D, Folstein M, Katzman R, Proce D, Stadlan EM (1984) Clinical diagnosis of Alzheimer's disease. Neurology 34:939-944

Miller SA, Dykes DD, Polesky HF (1988) A simple salting out procedure for extracting DNA from human nucleated cells. Nucl Acid Res 16:1215-1220

Poort SR, Rosendaal FR, Reitsma PH, Bertina RM (1996) A common genetic variation in the $3^{\prime}$-untranslated region of the prothrombin gene is associated with elevated plasma prothrombin levels and an increase in venous thrombosis. Blood 88:3698-3703

Rees DC, Liu YT, Cox MJ, Elliott P, Wainscoat JS (1997) Factor V Leiden and thermolabile methylenetetrahydrofolate reductase in extreme old age. Thromb Haemost 78:1357-1359
Ridker PM, Hennekens CH, Lindpainter K, Stampfer MJ, Eisenberg PR, Miletich JP (1995) Mutation in the gene coding for coagulation factor $\mathrm{V}$ and the risk of myocardial infarction, stroke, and venous thrombosis in apparently healthy men. N Engl J Med 332:912-917

Rosendaal FR, Koster T, Vandenbroucke JP, Reitsma PH (1995) High risk of thrombosis in patients homozygous for factor $\mathrm{V}$ Leiden (activated protein $\mathrm{C}$ resistance). Blood 85:1504-1508

Sacchi E, Duca F, Franceschi C, Mari D (1999) Prothrombin gene mutation (G20210A) in healthy centenarians. Thromb Haemost 81:990-991

Seshadri S, Fitzpatrick AL, Ikram MA et al (2010) Genomewide analysis of genetic loci associated with Alzheimer disease. JAMA 303:1832-1840

Zlokovic BV (2008) The blood-brain barrier in health and chronic neurodegenerative disorders. Neuron 57:178-201 